\title{
KFN-Studie zu sexuellem Missbrauch durch katholische Geistliche \\ Eine quantitative und qualitative Befragung von Betroffenen
}

Sandra Fernau, Deborah F. Hellmann

\section{Einleitung}

Nachdem das geplante Forschungsprojekt zwischen dem Kriminologischen Forschungsinstitut Niedersachsen (KFN) und dem Verband der Diözesen Deutschlands (VDD) zum sexuellen Missbrauch durch katholische Geistliche nicht in der ursprünglich geplanten Form durchgeführt werden konnte (siehe Pfeiffer, Mößle \& Baier, 2014; Kap. 1 in diesem Band), setzte das KFN auf eigene Initiative weitere Forschungsbemühungen um. Das Ziel der Forschung bestand insbesondere darin, die Entstehung und den Verlauf des sexuellen Missbrauchs aus Sicht der Betroffenen nachzuvollziehen, Umgangsweisen mit dem Erlebten zu rekonstruieren und psychosoziale Folgen der Taten zu analysieren. Zudem sollte dargestellt werden, wie sich die katholische Kirche gegenüber Täterinnen bzw. Tätern und Betroffenen verhalten hatte.

Hierzu wurden zwei Studien durchgeführt: zum einen eine quantitative Betroffenenbefragung, zum anderen eine qualitative Interviewstudie, in der Betroffene neben allgemeinen biografischen Erlebnissen ihre Missbrauchserfahrungen im Detail schilderten. Im Folgenden werden zunächst die Durchführung der quantitativen Erhebung und die Zusammensetzung der Betroffenenstichprobe sowie das verwendete Befragungsmaterial beschrieben. Daran schließt sich die Darstellung des methodischen Vorgehens bei der Erhebung und Auswertung im Rahmen der qualitativen Interviewstudie an.

\section{Die quantitative Betroffenenbefragung}

Um die bestehenden Forschungsdesiderate empirisch zu überprüfen (siehe Fernau, Treskow \& Stiller, 2014; Kap. 2 in diesem Band), wurden Be- 
troffene von sexuellem Missbrauch durch katholische Geistliche über verschiedene Kanäle aufgerufen, an der Betroffenenbefragung des KFN teilzunehmen. Die Betroffenen wurden im Vorfeld der Datenerhebung und zusätzlich in den Instruktionen zum Fragebogen darüber informiert, dass ihre Angaben anonym erfasst, absolut vertraulich behandelt und ausschließlich für wissenschaftliche Zwecke verwendet würden. Zusätzlich wurden sie mit Kontaktinformationen verschiedener Betroffenenverbände und Anlaufstellen für Betroffene sexuellen Missbrauchs versorgt, an die sie sich bei Bedarf wenden konnten. Die Studienteilnehmerinnen und -teilnehmer hatten die Möglichkeit, den Fragebogen entweder selbstständig aus dem Internet herunterzuladen und auszudrucken oder ihn per Post zu erhalten. Bei beiden Varianten war es möglich, den ausgefüllten Fragebogen kostenfrei an das KFN zurückzusenden. Durch dieses in der Betroffenenbefragung übliche Verfahren wurde keine repräsentative, sondern eine eher selektive Stichprobe Betroffener sexuellen Missbrauchs durch katholische Geistliche generiert (siehe z. B. Bergmann, 2011; LuegerSchuster, 2012). Um ihre Anonymität zu gewährleisten, wurden die Kontaktangaben der Befragten nach dem Ende der Erhebung vernichtet, sodass anhand der eingegangenen Fragebögen keine Rückschlüsse auf einzelne Betroffene möglich waren.

\section{II.1 Erhebungsmaterial}

Der Fragebogen zur Erfassung des sexuellen Missbrauchs an Minderjährigen durch katholische Geistliche umfasste insgesamt 28 Seiten. Nach einigen Vorbemerkungen zu den Zielen der Befragung und Hinweisen zum Ausfüllen des Fragebogens wurden in Teil A zunächst persönliche und soziodemografische Daten der Betroffenen erfragt. Teil B hatte die Kindheit und Jugend der Befragten zum Thema und in Teil $\mathrm{C}$ wurden spezifischen Angaben zum sexuellen Missbrauch erhoben. In Teil D wurden die Betroffenen zu der Zeit nach dem sexuellen Missbrauch befragt und in Teil E um Angaben zu ihrem derzeitigen Befinden gebeten. Einen Überblick über den Aufbau des Fragebogens bietet Abbildung 1. 


\begin{tabular}{|c|c|c|c|c|c|}
\hline \multicolumn{6}{|c|}{ Beiblatt mit Kontaktinformationen von Betroffenenverbänden } \\
\hline $\begin{array}{l}\text { Vorbemer- } \\
\text { kungen und } \\
\text { Ausfüll- } \\
\text { hinweise }\end{array}$ & $\begin{array}{l}\text { Teil A: } \\
\text { Angaben zur } \\
\text { Person }\end{array}$ & $\begin{array}{l}\text { Teil B: } \\
\text { Angaben zu } \\
\text { Kindheit und } \\
\text { Jugend }\end{array}$ & $\begin{array}{l}\text { Teil C: } \\
\text { Angaben zum } \\
\text { sexuellen } \\
\text { Missbrauch }\end{array}$ & $\begin{array}{l}\text { Teil D: } \\
\text { Angaben zur } \\
\text { Zeit nach } \\
\text { dem Miss- } \\
\text { brauch }\end{array}$ & $\begin{array}{l}\text { Teil E: } \\
\text { Angaben } \\
\text { zum derzeiti- } \\
\text { gen Befinden }\end{array}$ \\
\hline
\end{tabular}

Abbildung 1. Aufbau des Fragebogens der quantitativen Betroffenenbefragung

Zur statistischen Auswertung war der Fragebogen größtenteils im Multiple-Choice-Format aufgebaut, bot durch die Antwortoption "Sonstiges“ aber ebenfalls Raum für individuelle Erfahrungen und Anmerkungen. Darüber hinaus wurden einige offene Fragen ergänzt, um dem individuellen Missbrauchsgeschehen besser gerecht werden zu können. In den einleitenden Bemerkungen zum Fragebogen erhielten die Betroffenen erneut den Hinweis, dass die Teilnahme an der Studie freiwillig sei und ihre Anonymität strikt gewahrt werde. Zudem wurden sie noch einmal auf das anliegende separate Beiblatt mit Kontaktinformationen verschiedener Betroffenenverbände verwiesen und ihnen wurden die Kontaktinformationen der Projektleiterin mitgeteilt für den Fall, dass bei der Bearbeitung des Fragebogens Unklarheiten aufträten.

\section{Teil A: Persönliche und soziodemografische Angaben}

Um mehr über die soziodemografischen Hintergründe der Betroffenen zu erfahren, wurden sie zunächst nach ihrem Geburtsjahr, Geschlecht und Geburtsland gefragt. Mit Blick auf ihre Wohnsituation wurden die Befragten um Angaben zur Größe ihres aktuellen Wohnorts, zu ihrer konkreten Wohnsituation und zu der Anzahl an Personen, die mit ihnen im selben Haushalt lebten, gebeten. Die Antworten auf die Frage nach der aktuellen Wohnsituation in der Kategorie Sonstiges konnten von den Betroffenen näher spezifiziert werden und wurden für die Auswertung in Kategorien zusammengefasst (z. B. ,in einer Wohngemeinschaft“). Zudem benannten die Befragungsteilnehmerinnen und -teilnehmer ihren Familienstand, ihren höchsten Abschluss und ihre aktuelle Erwerbssituation. Die Antworten auf die Frage nach der aktuellen Erwerbssituation in der Kategorie Sonstiges konnten von den Betroffenen ebenfalls noch näher spezifiziert werden und wurden für die Auswertung in Kategorien zusammengefasst (z. B. ,arbeitsunfähig / Erwerbsminderungsrente"). 


\section{Teil B: Angaben zur Kindheit und Jugend}

Im nächsten Abschnitt des Fragebogens ging es um Erfahrungen aus der Kindheit und Jugend der Betroffenen. Dazu wurden sie gebeten, an die Zeit bis zu ihrem 16. Lebensjahr zurückzudenken. Zunächst wurde das elterliche Erziehungsverhalten mit sieben ausgewählten Items aus den Conflict Tactics Scales (Straus, 1979) erhoben, welche um vier eigene Items ergänzt wurden (vgl. Wetzels, 1997; siehe Abbildung 2). Damit umfasste dieser Bereich sowohl positive elterliche Zuwendung als auch elterliche Gewalt.

\begin{tabular}{|c|c|c|c|c|c|}
\hline $\begin{array}{l}\text { Eltern erziehen Ihre Kinder in sehr unter } \\
\text { sind einige Verhaltensweisen von Eltern b } \\
\text { wie oft Ihre Eltern oder die Personen, von } \\
\text { sich Ihnen gegenüber so verhalten haben. }\end{array}$ & & aur & lich & $\begin{array}{l}\text { Fol: } \\
\text { jeder } \\
\text { gen w }\end{array}$ & $\begin{array}{l}\text { en } \\
\text { e an, } \\
\text { en, }\end{array}$ \\
\hline $\begin{array}{l}\text { Meine Eltern / Erziehungspersonen haben } \\
\text {... }\end{array}$ & Nie & Selten & $\begin{array}{l}\text { Manch- } \\
\text { mal }\end{array}$ & Häufig & $\begin{array}{l}\text { Sehr } \\
\text { häufig }\end{array}$ \\
\hline $\begin{array}{l}\text { mich gelobt, wenn ich etwas besonders gut ge- } \\
\text { macht hatte. }\end{array}$ & $\square$ & $\square$ & $\square$ & $\square$ & $\square$ \\
\hline $\begin{array}{l}\text { mich in den Arm genommen und mit mir ge- } \\
\text { schmust. }\end{array}$ & $\square$ & $\square$ & $\square$ & $\square$ & $\square$ \\
\hline $\begin{array}{l}\text { mir ruhig erklärt, wenn ich etwas falsch ge- } \\
\text { macht hatte. }\end{array}$ & $\square$ & $\square$ & $\square$ & $\square$ & $\square$ \\
\hline mich getröstet, wenn ich traurig war. & $\square$ & $\square$ & $\square$ & $\square$ & $\square$ \\
\hline $\begin{array}{l}\text { mir etwas geschenkt bzw. mir etwas Besonderes } \\
\text { erlaubt, wenn ich etwas besonders gut gemacht } \\
\text { hatte. }\end{array}$ & $\square$ & $\square$ & $\square$ & $\square$ & $\square$ \\
\hline mit einem Gegenstand nach mir geworfen. & $\square$ & $\square$ & $\square$ & $\square$ & $\square$ \\
\hline mich hart angepackt oder gestoßen. & $\square$ & $\square$ & $\square$ & $\square$ & $\square$ \\
\hline mir eine runtergehauen. & $\square$ & $\square$ & $\square$ & $\square$ & $\square$ \\
\hline $\begin{array}{l}\text { mich mit der Faust geschlagen, getreten oder } \\
\text { mich gebissen. }\end{array}$ & $\square$ & $\square$ & $\square$ & $\square$ & $\square$ \\
\hline $\begin{array}{l}\text { mich mit einem Gegenstand geschlagen oder zu } \\
\text { schlagen versucht. }\end{array}$ & $\square$ & $\square$ & $\square$ & $\square$ & $\square$ \\
\hline mich geprügelt, zusammengeschlagen. & $\square$ & $\square$ & $\square$ & $\square$ & $\square$ \\
\hline
\end{tabular}

Abbildung 2. Befragungsmaterial zu elterlichem Erziehungsverhalten in Anlehnung an Straus (1979) und Wetzels (1997)

Des Weiteren hatten die Betroffenen die Möglichkeit anzugeben, ob sie in ihrer Kindheit oder Jugend zumindest zeitweise in einem Heim gelebt hatten. Die Personen mit Heimerfahrung in ihrer Kindheit oder Jugend wur- 
den im Anschluss mit sieben Items um Angaben zu der Atmosphäre und den Erziehungsmethoden gebeten, die im Heim geherrscht hatten (siehe Abbildung 3).

\begin{tabular}{|l|llllll|}
\hline \multicolumn{7}{|l|}{ Inwiefern treffen die folgenden Aussagen auf Sie zu? } \\
\hline $\begin{array}{l}\text { In der Zeit, während ich in einem } \\
\text { Heim gelebt habe ... }\end{array}$ & Nie & Selten & $\begin{array}{c}\text { Manch }- \\
\text { mal }\end{array}$ & Häufig & $\begin{array}{c}\text { Sehr } \\
\text { häufig }\end{array}$ \\
\hline herrschte eine liebevolle Atmosphäre. & $\square$ & $\square$ & $\square$ & $\square$ & $\square$ \\
\hline $\begin{array}{l}\text { wurde ich respektvoll von den Erwachsenen } \\
\text { behandelt. }\end{array}$ & $\square$ & $\square$ & $\square$ & $\square$ & $\square$ \\
\hline $\begin{array}{l}\text { hatten wir Kinder ein gutes Verhältnis zu- } \\
\text { einander. }\end{array}$ & $\square$ & $\square$ & $\square$ & $\square$ & $\square$ \\
\hline $\begin{array}{l}\text { war die Stimmung im Heim durch Angst } \\
\text { geprägt. }\end{array}$ & $\square$ & $\square$ & $\square$ & $\square$ & $\square$ \\
\hline $\begin{array}{l}\text { habe ich Demütigungen im Heim durch } \\
\text { Erwachsene erfahren. }\end{array}$ & $\square$ & $\square$ & $\square$ & $\square$ & $\square$ \\
\hline $\begin{array}{l}\text { wurde ich von den Erwachsenen im Heim } \\
\text { geschlagen. }\end{array}$ & $\square$ & $\square$ & $\square$ & $\square$ & $\square$ \\
\hline $\begin{array}{l}\text { wurde ich von anderen Kindern schlecht } \\
\text { behandelt. }\end{array}$ & $\square$ & $\square$ & $\square$ & $\square$ & $\square$ \\
\hline
\end{tabular}

Abbildung 3. Befragungsmaterial zur Atmosphäre und den Erziehungsmethoden im Heim

Als nächstes folgten zwei Items, um die Einbindung der Betroffenen in kirchliche Aktivitäten zu erfassen (,Ich war in die kirchliche Gemeindearbeit und -aktivität eingebunden“ und „Meine Freunde waren in die kirchliche Gemeindearbeit und -aktivität eingebunden"). Die daran anschließenden Fragen zur Bedeutung von Religion und Glauben der Betroffenen bezogen sich explizit auf den Zeitraum vor dem sexuellen Missbrauch. Dazu beantworteten die Befragungsteilnehmerinnen und -teilnehmer anhand einer vierstufigen Skala von „völlig unwichtig“ bis ,sehr wichtig“ zunächst, wie wichtig ihnen Religion und Glaube in der Zeit bis zum sexuellen Missbrauch waren. Im Anschluss daran wurde gefragt, wie oft die Betroffenen in dieser Zeit aus religiösem Anlass die Kirche besucht hatten und wie oft sie in dieser Zeit gebetet hatten. Die Häufigkeit der Kirchenbesuche wurde ebenso für Vater und Mutter der Betroffenen erfragt, allerdings mit der zusätzlichen Antwortoption „Ich weiß es nicht“. Darüber hinaus benannten die Befragten alle kirchlichen Aktivitäten, an denen sie in dieser Zeit teilgenommen hatten. Des Weiteren wurden die Befragungsteilnehmerinnen und -teilnehmer um Auskunft zur eigenen Religionszugehörigkeit sowie der von Vater und Mutter gebeten. Abschließend sollten 
die Betroffenen einschätzen, wie wichtig Religion und Glaube in der Erziehung durch die eigenen Eltern war.

\section{Teil C: Angaben zum sexuellen Missbrauch}

Im folgenden Fragebogenteil ging es um die näheren Umstände des sexuellen Missbrauchs und die Lebensumstände der Betroffenen zu dieser Zeit. Detaillierte Angaben zu denjenigen Itemformulierungen und Antwortformaten, die an dieser Stelle nicht wörtlich wiedergegeben werden, finden sich bei Hellmann, Dinkelborg, Wollinger \& Fernau (2014; Kap. 4 in diesem Band).

Zunächst wurden die Anzahl der Täterinnen bzw. Täter und der Zeitraum des sexuellen Missbrauchs im offenen Antwortformat festgestellt. Die Straftat des sexuellen Missbrauchs kann von ,leichteren“ Formen ohne Körperkontakt (z. B. Beobachten in Intimsituationen) über ,weniger schwere" Formen der Missbrauchshandlung (z. B. Versuch, Genitalien zu berühren) bis hin zu schwerem (z. B. Berührungen im Genitalbereich) und schwerstem Missbrauch (z. B. Penetration) reichen (z. B. Egle \& Abhary, 2005). Anhand von 13 ausgewählten Items wurden die spezifischen Missbrauchshandlungen in Anlehnung an Wetzels (1997) erfasst. Zudem wurde gefragt, welche Handlungen die Täterin bzw. der Täter darüber hinaus gegen die Betroffenen verübt hatte. Außerdem wurden das Erstviktimisierungsalter und das Alter der Täterin bzw. des Täters bei der ersten und der letzten Viktimisierung sowie das Alter der Betroffenen bei der letzten Missbrauchshandlung im offenen Format erfragt.

Das folgende Segment beleuchtete die Umstände des sexuellen Missbrauchs näher. Zunächst wurde erfasst, wie es beim ersten Mal zu einer sexuellen Missbrauchshandlung kam. Die anschließende Frage zielte auf den zeitlich-räumlichen Zusammenhang ab, in dem der Missbrauch in der Regel stattfand. Betroffene, die die Antwortoption Sonstiges benannten, hatten wiederum die Möglichkeit, ihre Angaben näher zu spezifizieren. Darüber hinaus sollten diejenigen Befragten, die bejaht hatten, dass die Täterin bzw. der Täter den Missbrauch in eine religiöse Handlung eingebettet hatte, diese Handlung näher beschreiben. Weiterhin wurde erhoben, ob die Betroffenen oder die Täterin bzw. der Täter während der sexuellen Missbrauchshandlung unter Alkohol- oder Drogeneinfluss standen und ob Fotos oder Videos vom Missbrauch angefertigt wurden. Zur Spezifikation des Täterprofils wurde die kirchliche Funktion der Täterin bzw. des Täters erfasst. Falls die Betroffenen in der nächsten Frage angegeben hatten, dass 
die Eltern mit der Täterin bzw. dem Täter bekannt waren, folgten sechs Items zur Intensität der Beziehung zwischen den Eltern und der Täterin bzw. dem Täter. Alle Befragten wurden danach um Angaben zu ihrem Lebensmittelpunkt zur Zeit des Missbrauchs sowie zur geschätzten Größe und religiösen Ausrichtung des Ortes gebeten, in dem sie zum Zeitpunkt des Missbrauchs gelebt hatten.

Als nächstes beantworteten die Betroffenen neun Items dazu, inwiefern die Täterin bzw. der Täter sie bedroht hätte für den Fall, dass sie über den Missbrauch sprächen (siehe Abbildung 4). Zudem war von Interesse, ob jemand bzw. wer von dem Verhalten der Täterin bzw. des Täters gewusst hatte.

\begin{tabular}{|c|c|c|c|}
\hline & $\begin{array}{c}\text { Trifft } \\
\text { nicht } z u\end{array}$ & $\begin{array}{c}\text { Trifft } \\
\text { teilweise zu }\end{array}$ & $\begin{array}{c}\text { Trifft } \\
\text { völlig zu }\end{array}$ \\
\hline $\begin{array}{l}\text { Der Täter hat mir gedroht, mir körperlich etwas anzu- } \\
\text { tun. }\end{array}$ & $\square$ & $\square$ & $\square$ \\
\hline Der Täter hat mir gedroht, sich selbst etwas anzutun. & $\square$ & $\square$ & $\square$ \\
\hline Der Täter hat mir schlimme Konsequenzen angedroht. & $\square$ & $\square$ & $\square$ \\
\hline $\begin{array}{l}\text { Der Täter hat mir gedroht, dass für ihn schlimme Kon- } \\
\text { sequenzen entstehen. }\end{array}$ & $\square$ & $\square$ & $\square$ \\
\hline Der Täter hat mir gedroht, dass mir niemand glaubt. & $\square$ & $\square$ & $\square$ \\
\hline $\begin{array}{l}\text { Der Täter hat mir gedroht, meiner Familie bzw. } \\
\text { Freunden etwas anzutun. }\end{array}$ & $\square$ & $\square$ & $\square$ \\
\hline Der Täter hat mir gedroht, dass Gott mich bestraft. & $\square$ & $\square$ & $\square$ \\
\hline $\begin{array}{l}\text { Der Täter hat mir gedroht, mir bestimmte Dinge zu } \\
\text { verwehren (bitte nennen): }\end{array}$ & $\square$ & $\square$ & $\square$ \\
\hline Der Täter hat mir Sonstiges angedroht (bitte nennen): & $\square$ & $\square$ & $\square$ \\
\hline
\end{tabular}

Abbildung 4. Befragungsmaterial zu potenziellen Drohungen der Täterin bzw. des Täters

Im Folgenden wurden potenzielle Zuwendungen der Täterin bzw. des Täters erfragt, welche sie oder er den Betroffenen vor, während oder nach dem Missbrauch zukommen lassen hatte (siehe Abbildung 5). Dabei bestand für die Betroffenen wiederum die Möglichkeit, für neun Items zu bewerten, ob das entsprechende Verhalten auf ihren Fall zutraf und wenn ja, auf welchen konkreten Zeitraum. 
Nun geht es darum, inwiefern Sie vom Täter in irgendeiner Form besondere Zuwendungen erhalten haben. Dabei interessiert uns auch der Zeitpunkt, zu dem Sie möglicherweise besondere Zuwen-dung(en) erhalten haben.

Bitte kreuzen Sie in jeder Zeile an, inwiefern die Aussage auf Sie zutrifft. Es ist auch möglich, in einer Zeile mehrere Kreuze zu setzen, wenn die Aussage auf mehrere Zeiträume zutrifft.

\begin{tabular}{|l|cccc|}
\hline & $\begin{array}{c}\text { Trifft } \\
\text { nicht } \\
z u\end{array}$ & $\begin{array}{c}\text { Trifft für die } \\
\text { Zeit } \\
\text { vor dem } \\
\text { Missbrauch } \\
\text { zu }\end{array}$ & $\begin{array}{c}\text { Trifft für die } \\
\text { Zeit } \\
\text { während } \\
\text { des Miss- } \\
\text { brauchs zu }\end{array}$ & $\begin{array}{c}\text { Triff für die } \\
\text { Zeit } \\
\text { nach Ende } \\
\text { des Miss- } \\
\text { brauchs zu }\end{array}$ \\
\hline $\begin{array}{l}\text { Der Täter hat mir bestimmte Privilegien im } \\
\text { Zusammenhang mit kirchlichen Aktivitä- } \\
\text { ten gestattet (z. B. Solosingen im Chor, } \\
\text { eingeschränkte Beichte) } \\
\text { (bitte nennen): }\end{array}$ & $\square$ & $\square$ & $\square$ & $\square$ \\
\hline $\begin{array}{l}\text { Der Täter hat mir bestimmte Privilegien im } \\
\text { Zusammenhang mit Freizeitaktivitäten ge- } \\
\text { stattet (z. B. länger aufzubleiben) } \\
\text { (bitte nennen): }\end{array}$ & $\square$ & $\square$ & $\square$ & $\square$ \\
\hline $\begin{array}{l}\text { Der Täter hat mir Geschenke gemacht / } \\
\text { Geld gegeben. }\end{array}$ & $\square$ & $\square$ & $\square$ & $\square$ \\
\hline $\begin{array}{l}\text { Der Täter hat mir Alkohol, Zigaretten oder } \\
\text { sonstige Drogen gegeben. }\end{array}$ & $\square$ & $\square$ & $\square$ & $\square$ \\
\hline $\begin{array}{l}\text { Der Täter hat mir zugehört oder mir be- } \\
\text { sondere Aufmerksamkeit geschenkt. }\end{array}$ & $\square$ & $\square$ & $\square$ & $\square$ \\
\hline $\begin{array}{l}\text { Der Täter hat mir erlaubt, bei ihm zu über- } \\
\text { nachten. }\end{array}$ & $\square$ & $\square$ & $\square$ & $\square$ \\
\hline $\begin{array}{l}\text { Der Täter hat mich mit in den Urlaub ge- } \\
\text { nommen. }\end{array}$ & $\square$ & $\square$ & $\square$ & $\square$ \\
\hline Der Täter hat meine Familie unterstützt. & $\square$ & $\square$ & $\square$ & $\square$ \\
\hline $\begin{array}{l}\text { Der Täter mir andere Zuwendungen erteilt } \\
\text { bitte nennen): }\end{array}$ & $\square$ & $\square$ & $\square$ & $\square$ \\
\hline
\end{tabular}

Abbildung 5. Befragungsmaterial zu potenziellen Zuwendungen der Täterin bzw. des Täters

Die darauffolgenden drei Fragen fokussierten auf die Endphase des sexuellen Missbrauchs. Zunächst wurde erfasst, wodurch das Ende des Missbrauchs herbeigeführt worden war. Da das Bewusstsein, sexuell misshandelt worden zu sein, häufig nicht gleich vorhanden ist (z. B. Sanderson, 1990), wurde erfragt, zu welchem Zeitpunkt und durch welche Situation dieses Bewusstsein induziert worden war. Der letzte Abschnitt dieses Fragebogensegments betraf die emotionalen, körperlichen und verhaltensrelevanten Folgen, die unmittelbar mit dem Missbrauch einhergingen. Detaillierte Schilderungen der Itemformulierungen und Antwortformate sind bei Hellmann, Dinkelborg und Fernau (2014; Kap. 6 in diesem Band) zu finden. 


\section{Teil D: Angaben zu der Zeit nach dem sexuellen Missbrauch}

Teil D des Fragebogens beinhaltete Fragen zu der Zeit nach dem sexuellen Missbrauch. Vor allem ging es um die Reaktionen aus dem sozialen und institutionellen Umfeld der Betroffenen (siehe auch Wollinger, Dinkelborg \& Baier, 2014; Kap. 7 in diesem Band) sowie die Entschädigung der Betroffenen (siehe auch Hellmann \& Bartsch, 2014).

Der erste Fragenkomplex bezog sich darauf, ob die Befragten jemals mit einer anderen Person über den Missbrauch gesprochen hatten. Falls dies der Fall war, sollten sie alle Personen benennen, denen sie vom Missbrauch erzählt hatten, sowie den ungefähren Zeitpunkt (siehe Wollinger et al., 2014; Kap. 7 in diesem Band). Die daran anschließenden neun Items dienten dazu, die Reaktionen der Personen zu erfassen, die von dem Missbrauch erfahren hatten. Diese Items thematisierten, ob den Betroffenen zugehört worden war; ob ihnen geglaubt worden war; ob ihnen geholfen worden war, das Erlebte zu verarbeiten; ob sie finanziell oder emotional unterstützt worden waren; ob sie abgewimmelt worden waren; ob nichts weiter passiert war; ob ihnen unterstellt worden war, die Täterin bzw. den Täter verführt zu haben oder verführen zu wollen und ob sie von Dritten öffentlich denunziert oder angegriffen worden waren. Darüber hinaus konnten die Befragten zusätzliche Reaktionen aufführen, die zuvor nicht benannt worden waren. Neben der Möglichkeit, im offenen Antwortformat Gründe für das anhaltende Schweigen über den Missbrauch zu ergänzen, wurden diejenigen Betroffenen, die nie mit jemandem über den Missbrauch gesprochen hatten, anhand der folgenden 16 Items nach ihren Gründen befragt:

- Weil ich das für mich allein geklärt habe.

- Weil es mir nichts bringen würde.

- Weil ich niemanden kenne, dem ich es erzählen könnte.

- Weil mir niemand glauben würde.

- Weil es niemand verstehen würde.

- Weil ich nicht darüber sprechen kann / möchte.

- Weil ich mich schuldig dafür fühl(t)e, was passiert ist.

- Weil es nicht so schlimm war.

- Weil ich das Ganze einfach nur vergessen möchte.

- Weil ich nicht möchte, dass dem Täter etwas passiert.

- Weil es für meine Familie eine große Belastung (gewesen) wäre.

- Weil ich Angst vor dem habe, was der Täter mir angedroht hat.

- Weil ich Angst davor habe, dass es öffentlich wird. 
- Weil der Täter zur Kirche gehörte.

- Weil ich das Ganze auf sich beruhen lassen möchte.

- Weil alles in Gottes Hand liegt.

Die nächsten Fragenkomplexe dieses Befragungssegments hatten die Reaktionen von Kirche und Strafverfolgungsbehörden auf den sexuellen Missbrauch zum Inhalt. Dabei ging es darum, ob (und wenn ja, wann) der Missbrauch der Kirche oder den Strafverfolgungsbehörden gemeldet worden war, was die Gründe für bzw. gegen die offizielle Meldung des Erlebten waren und welche Reaktionen darauf gegebenenfalls folgten. Zudem war von Interesse, ob die Befragten anderen Betroffenen zu einer offiziellen Meldung eines erlebten sexuellen Missbrauchs bei der Kirche oder den Strafverfolgungsbehörden raten würden. Zusätzlich wurde die Zufriedenheit mit der Behandlung durch die Kirche bzw. die Strafverfolgungsbehörden erfragt (siehe auch Wollinger et al., 2014; Kap. 7 in diesem Band).

Die nächsten vier Fragen in Abschnitt D betrafen potenziell bestehende Entschädigungsansprüche im Sinne des Opferentschädigungsgesetzes (OEG; siehe Hellmann \& Bartsch, 2014). Diesbezüglich wurde zunächst festgestellt, ob bzw. durch wen die Betroffenen in der Vergangenheit über ihren Anspruch auf Entschädigung im Sinne des OEG informiert worden waren. Zudem wurden die Betroffenen gebeten zu beantworten, ob sie oder eine andere Person bzw. Organisation einen Antrag nach dem OEG gestellt hatten. Wenn ein OEG-Antrag gestellt worden war, erhielten diese Befragungsteilnehmerinnen und -teilnehmer eine Liste mit zehn Aussagen bezüglich des OEG-Verfahrens, die sie anhand einer vierstufigen Skala bewerten sollten. Abschließend wurden diejenigen Betroffenen, die einen Antrag nach dem OEG gestellt hatten bzw. hatten stellen lassen, gebeten, den Verfahrensausgang in einem offenen Antwortformat zu beschreiben.

Schließlich sollten alle Befragten beantworten, welche Entschädigungsmaßnahme(n) sie sich im Nachhinein gewünscht hätten. Die Antwortoptionen beinhalteten die Entschuldigung und Wiedergutmachung durch die Täterin bzw. den Täter oder die katholische Kirche, finanzielle Entschädigungen, Beratung oder Therapie für die Betroffenen oder die Täterin bzw. den Täter, Täter-Opfer-Ausgleich sowie gerichtliche Strafen. Zudem bestand die Möglichkeit, dass die Betroffenen angaben, dass sie sich gar nichts im Nachhinein gewünscht hätten und dass sie andere Maßnahmen angaben, die nicht in den vorgegebenen Antwortoptionen enthalten waren.

Die letzte Frage dieses Abschnitts erfasste, ob die Befragten zum Erhebungszeitpunkt Kenntnis davon hatten, ob auch andere Kinder oder Ju- 
gendliche von derselben Täterin bzw. demselben Täter sexuell missbraucht worden waren. Diese Frage war ebenfalls von allen Befragungsteilnehmerinnen und -teilnehmern zu beantworten.

\section{Teil E: Das derzeitige Befinden}

Der letzte Fragebogenteil bezog sich auf das aktuelle Befinden der Betroffenen sowie ihren Umgang mit dem sexuellen Missbrauch und ihre derzeitige Einstellung zur Kirche und zum Glauben. Zunächst wurde die aktuelle Bedeutung von Religion und Glaube für das persönliche Leben thematisiert (siehe Hellmann et al., 2014; Kap. 6 in diesem Band). Parallel zu den Fragen in Abschnitt B, die sich auf den Zeitraum vor dem sexuellen Missbrauch bezogen, wurden die Betroffenen konkret danach gefragt, wie wichtig ihnen persönlich Religion und Glaube heute wären. Zudem wurden sie befragt, wie oft sie heute aus religiösem Anlass die Kirche besuchten und wie oft sie heute beteten. Außerdem wurden die Betroffenen gebeten, alle kirchlichen Aktivitäten zu benennen, an denen sie in der heutigen Zeit teilnahmen. Zusätzlich wurde erhoben, ob die Befragungsteilnehmerinnen und -teilnehmer zum Erhebungszeitpunkt aus der katholischen Kirche ausgetreten waren und wenn ja, wann sie ausgetreten waren und ob sie einer anderen Religionsgemeinschaft beigetreten waren. Falls sich die Befragten nach ihrem Austritt aus der katholischen Kirche einer anderen Religionsgemeinschaft angeschlossen hatten, wurden sie gebeten, diese zu benennen.

Im Anschluss daran war von Interesse, wie die Betroffenen die mediale Debatte um den sexuellen Missbrauch im Jahr 2010 erlebt hatten. Dazu sollten fünf Items anhand einer vierstufigen Skala danach bewertet werden, inwiefern sie auf die Befragten persönlich zutrafen. Konkret sollte angegeben werden, ob es geholfen hatte, über den Missbrauch zu sprechen; ob das Bewusstsein entstanden war, kein Einzelfall zu sein; ob erst durch die mediale Debatte der Mut gefunden worden war, das Schweigen $\mathrm{zu}$ brechen und ob die mediale Position der Kirche als um Aufklärung bemüht bewertet wurde. Ebenso erhielten die Betroffenen die Möglichkeit anzugeben, ob und welche Aspekte ihrer Auffassung nach medial verzerrt dargestellt worden waren.

Die nächsten drei Fragenkomplexe hatten zum Ziel, die überdauernden psychischen Belastungen der Betroffenen einstufen zu können. Dazu wurde zum einen die Posttraumatic Stress Disorder Checklist (PCL-C; Teegen, 1997; siehe auch Höcker \& Mehnert, 2012) zur Erfassung posttrau- 
matischer Belastungsstörungen (PTBS) eingesetzt. Zum anderen wurden mithilfe der Subskalen „Somatisierung“ (sieben Items), „Psychotizismus“ (fünf Items), „Paranoides Denken“ (fünf Items), „Phobische Angst“ (fünf Items) und „Depressivität“ (sechs Items) des Brief Symptom Inventory (BSI; Derogatis \& Melisaratos, 1983; Franke, 2000) Symptome physischer und psychischer Beeinträchtigungen betrachtet. Darüber hinaus wurde der Fragebogen $R S-11$ eingesetzt, der die individuelle Resilienz misst, also die Fähigkeit, schwierige Lebenssituationen ohne anhaltende Beeinträchtigung $\mathrm{zu}$ überstehen (Schumacher, Leppert, Gunzelmann, Strauß \& Brähler, 2005). In diesem Zusammenhang beurteilten die Betroffenen elf Items anhand einer siebenstufigen Skala mit den Ankerpunkten ,trifft gar nicht zu“ bis „trifft völlig zu“ (siehe Schumacher et al., 2005). Ein Beispielitem lautet „Ich kann mich überwinden, Dinge zu tun, die ich eigentlich nicht machen will." Details zu diesen Erhebungsinstrumenten werden bei Hellmann et al., 2014 (Kap. 6 in diesem Band) berichtet.

Zum Abschluss der Befragung wurde den Betroffenen Platz für persönliche Anmerkungen eingeräumt. Hier konnten sie beispielsweise weitere relevante Aspekte der sexuellen Missbrauchserfahrung vermerken und wünschenswerte bzw. erforderliche Hilfsangebote zur Verarbeitung selbiger benennen.

\section{II.2 Stichprobenbeschreibung}

Insgesamt wurden zwischen März und Oktober 2013 die Daten von $N=113$ Betroffenen erfasst. Aufgrund der Möglichkeit, den Fragebogen über das Internet zu beziehen, konnte keine Rücklaufquote bestimmt werden. Aus den statistischen Analysen wurden die Angaben derjenigen Befragten ausgeschlossen, die zur Zeit der ersten sexuellen Missbrauchshandlung bereits volljährig waren. Dabei wurde die Volljährigkeit anhand der zum Zeitpunkt des Missbrauchs geltenden Bestimmungen festgelegt. Ebenso konnten Daten von Betroffenen nicht berücksichtigt werden, wenn der bzw. die benannte(n) Täterin(nen) bzw. Täter nicht eindeutig als katholische Geistliche zu identifizieren waren. Im Rahmen der vorliegenden Studie definieren wir ein Verhalten als ,sexuellen Missbrauch durch katholische Geistliche“ daher, wenn

- mindestens eine von 13 konkret vorgegebenen sexuellen Handlungsformen 
- zwischen einer minderjährigen Person

- und mindestens einem katholischen Geistlichen

- mindestens einmal stattgefunden hat.

Zur Gruppe der „katholischen Geistlichen“ gehören dabei neben den üblicherweise hierunter gefassten Priestern (sowie Kaplänen bzw. Vikaren), Diakonen, Priesteramtsanwärtern und männlichen Ordensangehörigen auch Ordensschwestern, die im Bereich der Deutschen Bischofskonferenz tätig waren bzw. sind.

Die in diesem Band berichteten quantitativen Ergebnisse beziehen sich daher auf eine Stichprobe von $N=104$ Betroffenen mit einem mittleren Alter von $M=56.14$ Jahren $(S D=10.33) .{ }^{1}$ Das Alter der Befragten variierte zwischen 34 und 79 Jahren. Besonders stark waren Personen der Altersgruppe 50 bis 69 Jahre vertreten. Der Anteil von Frauen unter den Befragten war mit $n=26(25.0 \%)$ deutlich geringer als der Anteil männlicher Befragter mit $n=77(74.0 \% ; 1.0 \%$ fehlende Werte, $n=1)$. Diese Geschlechterverteilung entspricht sowohl den Ergebnissen des Forschungsberichts der Universität Wien (Lueger-Schuster, 2012) als auch den Angaben im Abschlussbericht der deutschen Bischofskonferenz (Zimmer, Lappehsen-Lengler, Weber \& Götzinger, 2014). Die höchste Anzahl an Vorfällen sexuellen Missbrauchs durch katholische Geistliche geschah der deutschen Bischofskonferenz zufolge zwischen 1950 und 1970. Dies steht ebenfalls in Einklang mit der vorliegenden Stichprobenzusammensetzung, da die Personen der am stärksten vertretenen Alterskategorie (50 bis 69 Jahre) in den Jahren 1950 bis 1970 minderjährig waren.

Der Großteil der Befragten (94.2\%) wurde in Deutschland geboren, sechs Teilnehmerinnen bzw. Teilnehmer stammten aus deutschen Anrainerstaaten oder aus Italien, Kanada und Jugoslawien. Zum Zeitpunkt der Befragung lebte die Hälfte der Betroffenen eigenen Angaben zufolge in einer Großstadt mit mehr als 100000 Einwohnerinnen und Einwohnern (1.9\% fehlende Werte, $n=2$; siehe Tabelle 1$)$. Jeweils rund $11 \%$ der Befragungsteilnehmerinnen und -teilnehmer lebten aktuell in einer Stadt mit

1 Die Abkürzung $M$ steht für den Mittelwert (Durchschnitt, arithmetisches Mittel) aller Messwerte einer spezifischen Variable. In diesem Fall bezeichnet $M$ das durchschnittliche Alter aller Befragten. $S D$ bedeutet Standardabweichung (Streuung) und berechnet sich aus der Wurzel der Varianz (Summe der quadrierten Abweichungen der Messwerte vom Mittelwert $M$ relativiert an der Anzahl aller Messwerte). Vereinfacht gesagt bildet die Standardabweichung die Unterschiedlichkeit der einzelnen Messwerte $a b$. 
Einwohnerzahlen von 50000 bis 99999 bzw. 20000 bis 49 999. In kleinen Städten mit einer Einwohnerzahl zwischen 2000 und 20000 lebten zum Befragungszeitpunkt insgesamt $21.3 \%$ der Betroffenen, knapp $6 \%$ gaben einen Wohnort mit weniger als 2000 Einwohnerinnen und Einwohnern an.

Tabelle 1. Größe des aktuellen Wohnorts der Befragten $(N=104)$

Einwohnerzahl $<2000$

Einwohner zwischen 2000 bis 4999

Einwohner zwischen 5000 bis 19999

Einwohner zwischen 20000 bis 49999

Einwohner zwischen 50000 bis 99999

Einwohner zwischen 100000 bis 499999

Einwohnerzahl $>500000$

Keine Angabe
$5.8 \%(n=6)$

$8.7 \%(n=9)$

$12.5 \%(n=13)$

$10.6 \%(n=11)$

$10.6 \%(n=11)$

$29.8 \%(n=31)$

$20.2 \%(n=21)$

$1.9 \%(n=2)$

Die meisten Befragten lebten eigenen Angaben zufolge allein (41.3\%) oder mit einem Partner bzw. einer Partnerin und / oder eigenen Kindern zusammen (43.3\%). Drei Personen (2.9\%) berichteten, dass sie mit Eltern oder Geschwistern zusammenlebten, $8.7 \%$ der Betroffenen lebten in einer nicht näher spezifizierten Wohnsituation und vier Personen $(3.8 \%)$ waren in einer Wohngemeinschaft zuhause.

Tabelle 2. Aktuelle Wohnsituation der Befragten $(N=104)$

\begin{tabular}{lr}
\hline Ein-Personen-Haushalt & $38.5 \%(n=40)$ \\
Zwei-Personen-Haushalt & $33.7 \%(n=35)$ \\
Drei-Personen-Haushalt & $10.6 \%(n=11)$ \\
Vier-Personen-Haushalt & $11.5 \%(n=12)$ \\
Haushalt mit fünf oder mehr Personen & $4.8 \%(n=5)$ \\
Keine Angabe & $1.0 \%(n=1)$ \\
\hline
\end{tabular}

Die Anzahl der in einem Haushalt lebenden Personen betrug folglich in den meisten Fällen eins (38.5\%; $1.0 \%$ fehlende Werte, $n=1$; siehe Ta- 
belle 2) oder zwei (33.7\%). 10.6\% der Befragten lebten in einem DreiPersonen-Haushalt, $11.5 \%$ lebten in einem Vier-Personen-Haushalt und $4.8 \%$ lebten mit vier oder mehr anderen Personen zusammen. Die Befragten waren größtenteils verheiratet (41.3\%), nahezu zwei von zehn Befragten waren eigenen Angaben zufolge ledig, fast ein Drittel lebte zum Befragungszeitpunkt getrennt oder in Scheidung und knapp $4 \%$ waren verwitwet (siehe Tabelle 3). $8.7 \%$ der Befragungsteilnehmerinnen und -teilnehmer berichteten, dass sie sich in einer festen Partnerschaft befänden.

Tabelle 3. Familienstand der Befragten $(N=104)$

\begin{tabular}{lr}
\hline Verheiratet & $41.3 \%(n=43)$ \\
Getrennt lebend oder geschieden & $26.9 \%(n=28)$ \\
Ledig & $19.2 \%(n=20)$ \\
In fester Partnerschaft & $8.7 \%(n=9)$ \\
Verwitwet & $3.8 \%(n=4)$ \\
\hline
\end{tabular}

Möglicherweise hing die im bundesweiten Vergleich deutlich erhöhte Anzahl getrennt bzw. in Scheidung lebender befragter Personen (siehe z. B. https://www-genesis.destatis.de/genesis/online) mit der Missbrauchserfahrung zusammen. Denn Probleme in der Partnerschaft und Sexualität werden als dritthäufigste Folge von Missbrauchserfahrungen im kirchlichen Umfeld benannt (z. B. Zimmer et al., 2014).

Zum Bildungsniveau der Befragten ist hervorzuheben, dass sie mehrheitlich einen Hochschul- oder Fachhochschulabschluss aufwiesen (38.5 \%; siehe Tabelle 4). Im Vergleich enthielt die vorliegende Stichprobe damit zum Beispiel sowohl mehr Hochschul- bzw. Fachhochschulabsolventinnen und -absolventen als auch mehr Befragte ohne Abschluss (6.7\%) als die Stichprobe der von der Universität Wien durchgeführten Betroffenenbefragung (Lueger-Schuster, 2012). 15.4\% der Befragten erlangten einen Volks- oder Hauptschulabschluss, $12.5 \%$ die mittlere Reife oder den Realschulabschluss, $11.5 \%$ die Fachhochschulreife oder das Fachabitur und 10.6\% gaben das Abitur als höchsten Abschluss an (1.0\% fehlende Werte, $n=1$ ). 
Tabelle 4. Bildungsniveau der Befragten $(N=104)$

\begin{tabular}{lr}
\hline Hochschul- oder Fachhochschulabschluss & $38.5 \%(n=40)$ \\
Volks- oder Hauptschulabschluss & $15.4 \%(n=16)$ \\
Mittlere Reife oder Realschulabschluss & $12.5 \%(n=13)$ \\
Fachhochschulreife oder Fachabitur & $11.5 \%(n=12)$ \\
Abitur & $10.6 \%(n=11)$ \\
(Noch) keinen Abschluss & $6.7 \%(n=7)$ \\
Sonstiges & $3.8 \%(n=4)$ \\
Keine Angabe & $1.0 \%(n=1)$ \\
\hline
\end{tabular}

Der Großteil der Befragten (45.2 \%) befand sich zum Zeitpunkt der Befragung in einem Beschäftigungsverhältnis auf Vollzeit- oder Teilzeitbasis (siehe Tabelle 5). Aufgrund des erhöhten Durchschnittsalters der vorliegenden Stichprobe war es wenig überraschend, dass $41.3 \%$ der Befragten Rente oder Frührente bezogen. Da sexuelle Missbrauchserfahrungen häufig psychische Erkrankungen zur Folge haben, die mit Arbeitsunfähigkeit einhergehen können (z. B. Häuser \& Schiedermaier, 2005), ist der vergleichsweise hohe Anteil an Rentnerinnen und Rentnern möglicherweise auf die Missbrauchserfahrung zurückzuführen. 11.6\% der Betroffenen bezeichneten sich als arbeitsuchend oder nicht berufstätig und zwei Personen waren Hausfrauen bzw. Hausmänner.

Tabelle 5. Erwerbssituation der Befragten $(N=104)$

Berufstätig (Teilzeit / Vollzeit)

(Früh-)Rente / Pension / arbeitsunfähig /

Erwerbsminderungsrente

Nicht berufstätig / arbeitssuchend

Hausfrau / Hausmann
$45.2 \%(n=47)$

$41.3 \%(n=43)$

$11.6 \%(n=12)$

$1.9 \%(n=2)$

$\mathrm{Zu}$ ihrer Religionszugehörigkeit im Jugendalter befragt, gaben $90.4 \%$ der Personen die römisch-katholische Konfession an. Jeweils $4.8 \%$ der Befragten waren in ihrer Kindheit eigenen Angaben zufolge evangelisch bzw. hatten eine andere oder keine Konfession. Von den $n=94$ Befragten mit römisch-katholischer Konfession in ihrer Kindheit und Jugend waren bis zum Erhebungszeitpunkt $69.1 \%$ aus der katholischen Kirche ausgetre- 
ten $(1.1 \%$ fehlende Werte, $n=1$; siehe dazu auch Hellmann et al., 2014; Kap. 6 in diesem Band).

\section{Die qualitative Interviewstudie mit Betroffenen}

Mit der Realisierung der qualitativen Interviewstudie konnte bereits im Herbst 2011 begonnen werden. In drei Erhebungsphasen wurden im Rahmen der Untersuchung insgesamt 15 teilnarrative, leitfadengestützte Interviews mit erwachsenen weiblichen und männlichen Betroffenen sexuellen Missbrauchs durch katholische Geistliche durchgeführt. Dabei fanden drei Gespräche mit Frauen und zwölf mit Männern statt.

Die Interviews wurden mit einem Tonbandgerät aufgezeichnet und nach festen Transkriptionsregeln im Wortlaut (mit allen Pausen, Betonungen, Füllwörtern etc.) verschriftlicht. Es erfolgte eine Anonymisierung sämtlicher Namen, Daten und Orte im Zuge der Transkription, sodass keine Rückschlüsse auf die jeweilige Person möglich sind. Alle Befragten wurden vor den Gesprächen in einem Informationsblatt ausführlich über den Umgang mit ihren Äußerungen aufgeklärt und gaben ihre ,informierte Einwilligung“ (Hopf, 2000, S. 591) zur Interviewteilnahme und späteren Auswertung des Materials.

Mehrheitlich dauerten die Interviews zwischen eineinhalb und drei Stunden; im Durchschnitt gingen sie über einen Zeitraum von etwas mehr als zwei Stunden. Die Durchführung der Gespräche fand in unterschiedlichen Settings statt: entweder im KFN, also im institutionellen Umfeld der Interviewerin, oder in der Wohnung der Befragten und somit in deren privatem Umfeld. Die Erzählpersonen konnten zwischen beiden Orten wählen, um das Interview dort zu führen, wo sie sich am wohlsten und sichersten fühlen. Hiermit sollte zu einer möglichst entspannten Atmosphäre und einer für die Betroffenen weitestgehend komfortablen Situation beigetragen werden.

\section{III.1 Kontaktierung der Interviewten}

Die Studienteilnehmerinnen und -teilnehmer wurden in den drei Erhebungsphasen auf je unterschiedlichen Wegen gewonnen: Zum einen erfolgte eine Kontaktaufnahme durch die Betroffenen selbst über eine eigens hierfür eingerichtete E-Mail-Adresse, auf die unmittelbar nach dem Projektstart mit dem Verband der Diözesen Deutschlands (VDD) im Juli 2011 
auf der Homepage des KFN verwiesen wurde. Auf diese Weise bekundeten zwei der Interviewten ihr Interesse an der Mitwirkung bei der qualitativen Untersuchung. Mit ihnen fanden im Herbst 2011 Gespräche statt. Zum anderen leiteten die Missbrauchsbeauftragten eines Bistums im Frühjahr 2012 ein Anschreiben des KFN an ihnen bekannte, potenzielle Interviewpartnerinnen und -partner weiter, die sie im Zuge des Entschädigungsverfahrens der katholischen Kirche kontaktiert hatten. ${ }^{2}$ Hierauf meldeten sich 21 Personen, von denen anhand eines von ihnen zuvor ausgefüllten kurzen Fragebogens zehn für ein Interview ausgewählt wurden. Der Fragebogen enthielt soziodemografische Angaben der Betroffenen sowie allgemeine Informationen zu ihren Missbrauchserfahrungen. Die Interviewten der dritten Erhebungsphase wurden wie die Teilnehmerinnen und Teilnehmer an der quantitativen Befragung durch einen öffentlichen Aufruf des KFN in verschiedenen Medien gewonnen. Es wandten sich daraufhin seit Anfang 2013 insgesamt 16 Betroffene, die von sich aus ihre Bereitschaft zur Teilnahme an einem Interview mitteilten, über verschiedene Kanäle an das KFN. Hiervon wurden drei Männer als Gesprächspartner ausgewählt. Die Selektion basierte auf ihren schriftlichen und teils zusätzlich auch telefonischen Schilderungen und erfolgte nach theoretischen Gesichtspunkten, die sich im Verlauf der Analyse der zuvor geführten Interviews herauskristallisiert hatten.

\section{III.2 Samplingverfahren}

In Anlehnung an gängige qualitative Samplingstrategien zielte die Fallauswahl grundsätzlich auf eine Erschließung der im Untersuchungsgebiet enthaltenen Variationsbreite ab, um so die „Strukturiertheit des Phänomens und das Spektrum seiner Ausprägungen“ (Przyborski \& WohlrabSahr, 2010, S. 176) zu erfassen. Es wurde demnach eine falltypologische Repräsentation der Heterogenität des Feldes in Bezug auf das Erkenntnis-

2 Nach der öffentlichen Aufdeckung zahlreicher Missbrauchsvorfälle innerhalb der katholischen Kirche bzw. ihren institutionellen Einrichtungen wurden im Rahmen eines bundesweiten Entschädigungsverfahrens in allen 27 deutschen (Erz-)Bistümern Missbrauchsbeauftragte eingesetzt, bei denen Betroffene von sexuellem Missbrauch durch katholische Geistliche einen Antrag auf materielle „Leistungen in Anerkennung des Leids" einreichen konnten (Deutsche Bischofskonferenz, Pressemitteilung vom 02.03.2011; http://www.dbk.de/fileadmin/redaktion/diverse_downloads/Dossiers/20 11-028a-Leistungen.pdf; zuletzt abgerufen am: 29.11.2013). 
interesse angestrebt. Der Grundgedanke hierbei ist, dass eine breite Variation innerhalb des Samples eine empirisch begründete Theoriebildung auf der Grundlage des Fallmaterials ermöglicht, die auch bei einer geringeren Fallzahl Verallgemeinerungen zulässt (Kelle \& Kluge, 1999; Kleining, 1982; Kruse, 2014; Przyborski \& Wohlrab-Sahr, 2010).

In der vorliegenden Interviewstudie fand eine Kombination von zwei kontrastierenden Samplingverfahren statt, die in verschiedenen Phasen des Forschungsprozesses zum Einsatz kamen. Nachdem mit der Durchführung von zwei Interviews zu Beginn der Erhebung bereits erste Eindrücke vom zu erschließenden Feld gewonnen werden konnten, gestaltete sich der weitere Prozess der Fallauswahl wie nachstehend beschrieben:

Im Rahmen der zweiten Erhebungswelle orientierte sich das Sampling an Kriterien, die im Zuge der Literatur- und Materialsichtung bestimmt wurden, folgte also theoretisch begründeten Vorabfestlegungen. Mittels der im Voraus festgelegten Merkmalsausprägungen sollte eine Spanne von möglichst unterschiedlichen Fällen erhoben werden (Kruse, 2014; Przyborski \& Wohlrab-Sahr, 2010). Bei den in dieser Studie verwendeten Varianzmerkmalen geht es neben soziodemografischen Aspekten wie Alter, Geschlecht ${ }^{3}$ und Bildungsstand vor allem um forschungsthematisch spezifische Gesichtspunkte: So wurden für die Fallauswahl auch der jeweilige Missbrauchskontext sowie das Alter bei Beginn der sexuellen Übergriffe und der Zeitraum, in dem diese stattfanden, berücksichtigt. Hiermit sollte eine heterogene Zusammensetzung des Samples erreicht werden, um ein möglichst breites Spektrum an Erfahrungen, Deutungs- und Verarbeitungsmustern erschließen zu können.

Zur Vermeidung einer Fokussierung auf die bereits vorab definierten Kriterien bzw. die (Vor-)Annahme einer Bedeutung dieser in Hinblick auf die Produktion von forschungsrelevanten Unterschieden, kam am Ende der zweiten sowie insbesondere in der letzten Erhebungsphase eine weitere Samplingmethode hinzu, die den im Interviewmaterial selbst gefundenen Besonderheiten und Kontrasten mehr Rechnung trägt. Es handelt sich

3 Hinsichtlich der Kategorie Geschlecht wurde bei der Fallauswahl lediglich berücksichtigt, dass sowohl männliche als auch weibliche Personen interviewt werden und keine gleiche oder ähnliche Verteilung angestrebt. Der Anteil von weiblichen Befragten ist insofern deutlich geringer als der Anteil männlicher Teilnehmer. Dies spiegelt auch die häufigere Betroffenheit von Männern bzw. Jungen von sexuellen Übergriffen durch katholische Geistliche wider, die für das Forschungsfeld innerkirchlichen sexuellen Missbrauchs charakteristisch ist (z. B. Bundschuh, 2010; John Jay College, 2004; Zimmer et al., 2014). 
dabei um das durch Glaser und Strauss (1967) in Zusammenhang mit der Entwicklung der Grounded Theory begründete und verschiedentlich weiterentwickelte (siehe z. B. Strauss, 1998) Verfahren des Theoretical Sampling. Zentral für diese Vorgehensweise ist die dynamische, sukzessive Weiterentwicklung der Samplestruktur im Forschungsprozess nach theoretischen Aspekten, die sich im Verlauf der empirischen Analyse herauskristallisieren. Die zu untersuchenden Fälle werden also allmählich im Wechsel von Datenerhebung und -interpretation auf Basis eines fortwährenden Vergleichs ausgesucht. Dabei folgt die Auswahl dem Grundsatz einer minimalen und maximalen Kontrastierung, mittels derer die durch die Analyse des bereits erhobenen Materials gewonnenen Konzepte modifiziert und differenziert werden und eine Auslotung der Varianz im Untersuchungsgebiet stattfindet. Ziel ist es, im Feld keine ,theoretisch relevanten Ähnlichkeiten und Unterschiede“ (Kelle \& Kluge, 1999, S. 46) mehr zu finden, um eine theoretische Sättigung zu erreichen (Kelle \& Kluge, 1999; Przyborski \& Wohlrab-Sahr, 2010; Strauss, 1998).

Die Auswahl der letzten Fälle basierte demnach auf Erkenntnissen, die im Zuge der Interpretation der zuvor geführten Interviews herausgearbeitet und theoriebezogen weiterentwickelt worden waren. Im Mittelpunkt standen dabei religionssoziologische Überlegungen, die sich aus der Beobachtung des in den Narrativen in verschiedenen Formen stattfindenden Rekurses auf religiöse Semantiken, katholische Glaubensvorstellungen und -rituale ergaben. Dieser oftmals anzutreffende Rückgriff auf katholisch geprägte Interpretationsvorlagen, der die Deutung lebensgeschichtlicher Konflikt- und Krisenerfahrungen im Allgemeinen wie auch die des sexuellen Missbrauchs im Speziellen beeinflusst, sollte durch die gezielte Auswahl neuer Fälle weiter vertieft werden. Angestrebt wurde dabei, anhand minimal und maximal kontrastierender Fälle, Unterschiede in der Bezugnahme auf religiöse Inhalte im Umgang mit biografischen (Konflikt-)erfahrungen zu generieren. Die hierfür notwendige grobe Vorabeinschätzung potentieller Interviewinhalte wurde durch die schriftlichen und teils telefonischen Schilderungen der Betroffenen im Zuge ihrer Selbstmeldung beim KFN möglich.

\section{III.3 Samplebeschreibung}

Insgesamt wurden im Rahmen der qualitativen Untersuchung 15 teilnarrative, leitfadengestützte Interviews mit erwachsenen weiblichen und männlichen Betroffenen sexuellen Missbrauchs durch katholische Geistliche ge- 
führt. Die Altersspanne der Befragten zum Zeitpunkt des Interviews reicht von 37 bis 71 Jahren und beträgt im Durchschnitt 58 Jahre. Diesbezüglich bestehen Ähnlichkeiten zwischen der Samplezusammensetzung der qualitativen Studie und der Stichprobenzusammensetzung der quantitativen Befragung. Die Viktimisierungserfahrungen der Betroffenen liegen in beiden Untersuchungen bereits mehrere Jahrzehnte zurück. Die Kontexte, in denen die sexuellen Übergriffe stattfanden, sind heterogen: Es wurden Personen mit Missbrauchserfahrungen in geschlossenen Institutionen (Heimen und Internaten), in Bildungseinrichtungen sowie in allgemeinen kirchlichen und privaten Zusammenhängen interviewt. Alle berichteten, in den Einzelheiten sehr unterschiedlichen Missbrauchshandlungen fanden mit Körperkontakt statt. Erfolgten diese zunächst ohne Berührungen, so trat der Körperkontakt im Laufe der Zeit hinzu. Es wurden sexuelle Aktivitäten mit, vor und auch an den Betroffenen geschildert. Zum Zeitpunkt des Beginns der sexuellen Übergriffe waren die Studienteilnehmerinnen und -teilnehmer zwischen 4 und 17 Jahren alt; durchschnittlich entwickelte sich die Missbrauchsbeziehung ab dem elften Lebensjahr. Der sexuelle Missbrauch ging in den allermeisten Fällen über mehrere Jahre und erstreckte sich mit Blick auf das gesamte Sample über einen Zeitraum zwischen ca. einem halben Jahr und neun Jahren. ${ }^{4}$

\section{III.4 Erhebungsverfahren: Biografische, teilnarrative Leitfadeninter-} views

Als Erhebungsinstrument wurde ein biografisch angelegter, teilnarrativer Interviewleitfaden eingesetzt, der sich an der Konzeption ,teilnarrativer Leitfadeninterviews" von Helfferich (2009) und Kruse (2014) orientiert. Hierbei handelt es sich um eine Mischform zwischen dem klassischen „narrativen Interview“ (Lucius-Hoene \& Deppermann, 2002; Schütze, 1976, 1978, 1983), in dessen Mittelpunkt die Stimulierung einer Stegreiferzählung steht, und dem mit einem mehr oder minder vorgegebenen Fragenkatalog arbeitenden und deshalb stärker strukturierten (und strukturierenden) „Leitfadeninterview“ (Hopf, 1978; Merton, Fiske \& Kendall, 1956).

4 Weitere Ausführungen zur Samplezusammensetzung der qualitativen Interviewstudie, insbesondere in Hinblick auf die verschiedenen Kontexte, in denen die sexuellen Übergriffe stattfanden, enthält Kapitel 5 dieses Sammelbands (Fernau, 2014). 
Formaler Aufbau des Interviewleitfadens: Gesprächsgestaltung und -organisation im teilnarrativen Leitfadeninterview

Die zentrale Absicht der Gesprächsführung im teilnarrativen Leitfadeninterview besteht darin, „mit der Strukturierung immer wieder narrative Teilerzählungen zu generieren“ (Helfferich, 2009, S. 179). Es geht also darum, den Erzählpersonen trotz bestimmter thematischer Vorgaben das monologische Rederecht innerhalb dieser Fokussierungen zuzugestehen, um ihnen ausreichend Raum für eigene Relevanzsetzungen und Detaillierungen zu bieten. Die Gesprächsorganisation zielt darauf ab, durch offen formulierte Fragen und eine flexible Handhabung ihrer Reihenfolge den Interviewten möglichst wenig vorzugeben, sondern sie vielmehr zum freien Erzählen anzuregen und auf diese Weise selbstläufige Schilderungen zu generieren. Neben dem Bestreben, die Interventionen seitens der Interviewerin gering zu halten, wird der Grundsatz verfolgt, im Interviewverlauf so weit als möglich das Vokabular der Befragten zu adaptieren und damit nicht in ihre semantische Struktur einzugreifen. Das zugrunde liegende Prinzip lautet demnach: ,So offen und flexibel [...] wie möglich, so strukturiert wie aufgrund des Forschungsinteresses notwendig“" (Helfferich, 2009, S. 181).

Zur Umsetzung dieser Vorgaben fanden bei der Konzeption des Leitfadens insbesondere zweierlei Gesichtspunkte Berücksichtigung:

1. Der Leitfaden wurde so aufgebaut, dass er eine flexible Handhabung von Strukturierung und Offenheit in der Interviewsituation ermöglicht. Es wurden zwar bestimmte thematische Bereiche vorgegeben, jedoch fand eine Anpassung ihrer Reihenfolge an den jeweiligen Erzählverlauf der Interviewten statt. Gleiches galt für die den verschiedenen Themenblöcken zugeordneten (möglichen) inhaltlichen Nachfragen, deren Abfolge ebenfalls flexibel gehandhabt wurde. Zudem wurden diese zwar als Orientierungshilfe vor$\mathrm{ab}$ im Wortlaut notiert, die konkrete Frageformulierung allerdings spontan gewählt und dem jeweils spezifischen Sprachstil der Interviewten zur Stützung ihrer Gesamterzählung angeglichen.

2. Der kommunikative Einstieg in die lebensgeschichtliche Erzählung erfolgte durch offen formulierte Erzählaufforderungen (Leitfragen) - sowohl zu Beginn des Interviews in Hinblick auf die Kindheit und Jugend der Befragten als auch an späterer Stelle bezüglich ihres weiteren Lebensverlaufs. Mit diesen Erzählstimuli, die ein weit gefasstes Themenfeld recht vage eröffnen, erhielten die Interview- 
ten die Möglichkeit zur eigenstrukturierten Thematisierung und Positionierung auf inhaltlicher wie sprachlicher Ebene. Erst daraufhin wurden Fragen gestellt, die eine stärkere thematische Fokussierung aufweisen und somit eine Strukturierung und Steuerung des Erzählverlaufs nach sich ziehen, ohne jedoch eine schließende Wirkung zu entfalten (Brückenkopffragen, exmanente Nachfragen). Ein Rückgriff auf diese vorformulierten Fragen fand zur Einführung neuer Themen oder noch nicht benannter Aspekte statt, sofern die Erzählpersonen den jeweiligen thematischen Gesichtspunkt nicht von sich aus ansprachen. Viel Raum wurde vor allem für solche Fragen gelassen, die keine bzw. möglichst wenige Präsuppositionen oder inhaltliche Impulse, im Sinne von externen Relevanzsetzungen, beinhalten (Aufrechterhaltungsfragen, immanente Nachfragen). Es erhielten also jene Fragen in der Handhabung des Leitfadens eine besondere Bedeutung, welche die Interviewten bei einer selbstläufigen Darstellung unterstützen und die inhaltliche und sprachliche Entwicklung ihrer Schilderung nur wenig beeinflussen. Hierbei handelte es sich in erster Linie um Aufforderungen zur Detaillierung oder Konkretisierung bereits benannter oder in den geschilderten Zusammenhang gehörender Aspekte sowie um den Erzählgang vorantreibende, inhaltsleere Fragen. ${ }^{5}$

\section{Konstruktion des Interviewleitfadens}

Ebenso wie bei der Gesprächsführung im Interview eine Orientierung an der Konzeption teilnarrativer Leitfadeninterviews von Helfferich (2009) und Kruse (2014) stattfand, erfolgte auch die Leitfadenerstellung in Anlehnung an das von das Helfferich (2009) vorgeschlagene „SPSS-Prinzip“ (Helfferich, 2009, S. 182). Hinter den Buchstabenkürzeln verbergen sich die vier, in der Forschungspraxis zwar nacheinander ablaufenden, jedoch

5 Beispiele für derartige Fragen sind:

- Möchten Sie dazu noch mehr erzählen? Möchten Sie das noch näher / ausführlicher / genauer beschreiben? Gibt es sonst noch etwas? Was fällt Ihnen noch etwas ein, was Ihnen wichtig ist? Was verbinden Sie noch mit ...?

- Was meinen Sie damit (konkret)? Können Sie ein Beispiel für ... nennen? Wie war das für Sie?

- Und dann? Wie ging es dann weiter? 
trotzdem zirkulär aufeinander bezogenen Arbeitsschritte „Sammeln“, „Prüfen“, „Sortieren“ und „Subsumieren“ (Helfferich, 2009).

Ziel der ersten Phase (Sammeln) ist es, in einem offenen Brainstorming mit Personen aus unterschiedlichen thematischen und gegebenenfalls disziplinären Bezügen möglichst viele Fragen mit mehr oder weniger engem Bezug zum Forschungsfeld zusammenzutragen. Reflexionen bezüglich ihrer inhaltlichen und sprachlichen Eignung sollen dabei zunächst zurückgestellt werden. Vielmehr besteht die Absicht darin, während des Ideenfindungsprozesses in der Gruppe eine hohe ,assoziative Dynamik“ (Kruse, 2014, S. 236) zu entfalten, um anhand unterschiedlicher Perspektiven und Zugänge eine Fülle an verschiedenartigen Fragen zu generieren (Helfferich, 2009; Kruse, 2014).

Im vorliegenden Projekt erfolgte das Sammeln von Fragen im Rahmen eines Interviewworkshops innerhalb einer Arbeitsgruppe von fünf Personen aus unterschiedlichen Fachdisziplinen. Die Mitglieder waren dazu angehalten, in hohem Tempo und unter Ausklammerung etwaiger Bedenken, möglichst viele Fragen je separat auf Karteikärtchen zu notieren. Diese wurden unmittelbar nach der Notation reihum und ohne Kommentierung vorgelesen. Im Anschluss an das Brainstorming fand sodann gemeinsam eine erste Besprechung und grobe Strukturierung der Fragen statt. Die Gruppenarbeit erwies sich durch die Produktion einer Vielfalt von unterschiedlich ausgerichteten Fragen als sehr ergiebig. Insgesamt umfasste die auf diese Weise entstandene Frageliste knapp 50 Fragen unterschiedlichster Art.

Im zweiten Schritt (Prüfen) findet eine Überprüfung der Fragen in Hinblick auf ihre Geeignetheit statt. Dabei wird die Frageliste insbesondere unter Aspekten des eigenen Vorwissens und der Offenheit durchgearbeitet. Im Zuge dessen kommen mehrere Prüffragen zum Einsatz, anhand derer eine Revision der Frageliste erfolgt. Zuerst werden alle Faktenfragen (als reine Informationsfragen) gestrichen. Danach werden diejenigen Fragen eliminiert, die aufgrund ihrer Formulierung eine tendenziell schließende Wirkung entfalten, also eher keine offenen Antworten oder Erzählungen erzeugen und damit dem Prinzip der Hörerorientierung zuwiderlaufen. Weitere Prüffragen erkunden die in Frageformulierungen implizierten Vorannahmen und Erwartungen. Hier ist insbesondere von Belang, was bereits bekannt ist und, ob das Erfragte lediglich dazu dient, die Interviewten dazu zu bringen, das eigene Vorwissen zu bestätigen. In diesen Zusammenhang fallen auch Überlegungen darüber, was möglicherweise Überraschendes bzw. den eigenen Vorannahmen Widersprechendes mitgeteilt werden könnte bzw. ob die Fragen überhaupt so offen formuliert 
sind, dass sie den Interviewten die Möglichkeit bieten, Unerwartetes zu berichten. All jene Fragen, die ausschließlich auf Präsuppositionen rekurrieren, werden aus der Liste gestrichen. Ferner werden Fragen nach abstrakten Zusammenhängen entfernt, welche die Tendenz aufweisen, weniger auf das Relevanzsystem der Befragten einzugehen, sondern eher theoretische Rahmenkonzepte an sie heranzutragen. Hierunter fallen vor allem Fragen, die auf die Überprüfung theoretischer Annahmen abzielen und oftmals durch das zugrundeliegende Forschungsinteresse motiviert werden. Deshalb handelt es sich bei ihnen mehr um Auswertungs- als um Interviewfragen (Helfferich, 2009; Kruse, 2014).

Die Überprüfung der Frageliste erfolgte in der vorliegenden Studie nach dem Interviewworkshop im Wechselspiel zwischen methodischen Überlegungen und einer weiteren Literatursichtung zum Themenfeld des sexuellen Missbrauchs durch katholische Geistliche einerseits, zu religionssoziologischen Zusammenhängen andererseits. Dies diente der Präzisierung und Konkretisierung des Forschungsinteresses, gleichzeitig aber auch der Vergegenwärtigung von theoriegeleiteten Vorannahmen und Intentionen im Interview. Daraufhin wurden die Fragen der ersten Auflistung bezüglich ihrer Eignung, die Interviewten zum Erzählen anzuregen und dabei möglichst offen und in Hinblick auf vorab Angenommenes indirekt zu sein, kontrolliert. Die Revision betraf weit mehr als die Hälfte aller Fragen der ersten Auflistung. Dabei wurden die meisten von ihnen ersatzlos gestrichen, bei manchen die Formulierung korrigiert und einige in Stichworte $\mathrm{zu}$ verschiedenen inhaltlichen Aspekten überführt. Neben der Überarbeitung von bestehenden Fragen wurden auch Ergänzungen und Differenzierungen vorgenommen.

Im nächsten Arbeitsschritt (Sortieren) werden die übrig gebliebenen Fragen und Stichworte inhaltlich und formal geordnet. Die Sortierung kann bei biografisch angelegten Interviews sowohl nach einer zeitlichen Dimension als auch nach forschungsspezifischen, inhaltlich-thematischen Perspektiven erfolgen. Sie dient der Bündelung von jeweils aufeinander bezogenen bzw. aneinander anschließenden Fragen unter relevante Kategorien (Helfferich, 2009; Kruse, 2014).

Da die vorliegenden Interviews auf eine lebensgeschichtliche Erzählung der Studienteilnehmerinnen und -teilnehmer abzielten, bot sich hier ein Sortieren nach einer zeitlichen Abfolge an. Zugleich lag eine Zuordnung von inhaltlichen Aspekten bzw. Themenbereichen zu den jeweiligen biografischen Verläufen nahe, weshalb beide Sortierungsweisen miteinander kombiniert wurden. 
Der letzte Schritt (Subsumieren) besteht nun darin, für die zuvor sortierten Fragen jeweils eine möglichst offene Erzählaufforderung zu finden, unter die sich die auf Einzelaspekte bezogenen Nachfragen und Stichworte einordnen lassen. Entscheidend ist hierbei, einen möglichst erzählgenerierenden und wenige Präsuppositionen enthaltenden Impuls $\mathrm{zu}$ wählen (Helfferich, 2009; Kruse, 2014). Im Zuge des Subsumierens erhält der Leitfaden seine Form: Die einzelnen Fragen und Stichpunkte werden in inhaltliche Aspekte, konkrete Nachfragen und Aufrechterhaltungsfragen unterschieden und unter die zur Einführung in die verschiedenen Themengebiete gewählten Erzählaufforderungen eingeteilt.

Bei dieser Studie wurden, wie oben bereits erwähnt, als einführende Erzählimpulse zwei allgemein gehaltene biografische Leitfragen verwendet, an die sich pro Themenbereich je eine inhaltlich stärker fokussierende Brückenkopffrage anschließen. Hierunter wurden jeweils die verschiedenen Stichworte zu inhaltlichen Aspekten, exmanente und immante Nachfragen sowie Aufrechterhaltungsfragen subsumiert.

\section{Themenübersicht und Fragen des Interviewleitfadens}

Nach der Skizzierung des formalen Aufbaus und der zentralen Arbeitsschritte bei der Konstruktion des Interviewleitfadens wird abschließend kurz auf seinen thematischen Aufbau eingegangen und das konkrete Vorgehen erläutert. Ausgehend von zwei diachronen Leitfragen zur Kindheit und Jugend sowie dem weiteren Lebensverlauf der Befragten gliedert sich der Interviewleitfaden in fünf Themenbereiche, die je mit offen formulierten Fragen (Brückenkopffragen) eingeleitet wurden: 1. die familiäre Erziehung und Sozialisation, 2. die religiöse Erziehung und Sozialisation sowie die in diese Zeit fallenden sexuellen Missbrauchserfahrungen, 3. die von den Betroffenen geführten Partnerschaften, 4. die Deutung und Verarbeitung des sexuellen Missbrauchs, 5. die Bedeutung von Religiosität und Gläubigkeit für die Interviewten sowie ihr Verhältnis zur katholischen Kirche.

Zur Veranschaulichung der Vorgehensweise im Interview sollen nachstehend einige Fragen exemplarisch angeführt werden. Da sie nur dann gestellt wurden, wenn die Betroffenen in ihrer lebensgeschichtlichen Erzählung nicht von alleine auf die jeweiligen Themengebiete zu sprechen kamen, wurden sie nicht in allen Interviews aufgegriffen. Vielmehr konnte immer wieder auf einige der Fragen verzichtet werden, da fast alle Interviewten sehr selbstläufige und umfassende Schilderungen produzierten, in 
denen viele thematisch relevante Aspekte bereits enthalten waren. Lediglich der erste Erzählstimulus wurde allen Personen gestellt. Die verschiedenen verwendeten Fragen differierten in ihren Formulierungen von Interview zu Interview nur minimal; der erste Erzählstimulus wurde allen Befragten im gleichen Wortlaut gestellt.

Diese Eingangserzählaufforderung lautete: „Als erstes möchte ich gerne mehr darüber erfahren, wie Sie so aufgewachsen sind. Bitte erzählen Sie mir doch all das, was Ihnen dazu so einfällt." Hieran schlossen je als Einleitung in die verschiedenen Themengebiete die nachstehenden zwei Brückenkopffragen an: „Wie war denn so das alltägliche Leben in Ihrer Familie?" in Hinblick auf die familiäre Erziehung und Sozialisation der Befragten, bezüglich ihrer religiösen Erziehung und Sozialisation sowie des in diesem Kontext erlebten sexuellen Missbrauchs die Frage: ,Wie haben Sie die Zeit im Internat / Heim / in der kirchlichen Gemeinde / als Messdienerin bzw. Messdiener erlebt?"

Als zweite Leitfrage diente der Stimulus „Nun interessiert mich, wie Ihr Leben denn so weiterverlaufen ist. Bitte erzählen Sie mir doch, was Ihnen dazu so einfällt." Zur Hinführung in die forschungsrelevanten thematischen Bereiche wurde sodann beispielsweise die Brückenkopffrage „Es gibt im Volksmund das Sprichwort, Die Zeit heilt alle Wunden'. Wie stehen Sie zu dieser Aussage?" verwendet. Hiermit sollte eine Schilderung in Hinblick auf die Deutung und Verarbeitung des sexuellen Missbrauchs generiert werden. Daran knüpften unter anderem zwei inhaltliche Nachfragen an, die sich konkreter mit der Beziehung zum Täter $^{6}$ sowie der Selbstdeutung der Betroffenen in Bezug auf ihre Viktimisierungserfahrungen befassen. Es handelte sich zum einen um die Bitte, sich das folgende Szenario auszumalen „Wenn Sie sich vorstellen, dass Sie dem Täter einen Brief schreiben, was möchten Sie darin gerne sagen?", zum anderen um die Frage „Was verbinden Sie mit dem Wort ,Opfer'?". Die Brückenkopffrage „Derzeit wird ja recht viel über die katholische Kirche berichtet. Was empfinden Sie in solchen Momenten?" zielte auf die Bedeutung von (katholischer) Religiosität für die Interviewten sowie ihr Verhältnis zur katholischen Kirche ab. Unter diesem Themenbereich findet sich außerdem zum Beispiel die Frage „Welche Bedeutung hat der Glaube an Gott für Sie heute?"“, mit welcher die alltagspraktische Rolle von Glaubensvor-

6 Da in der Interviewstudie sämtliche Täter männlichen Geschlechts sind, wird bei allen Aussagen, die sich ausschließlich auf die qualitative Untersuchung beziehen, die maskuline Form verwendet. 
stellungen (auch unabhängig vom Eingebundensein in kirchliche Strukturen) eruiert werden sollte.

\section{III.5 Auswertungsverfahren}

Die Auswertung der transkribierten Interviews erfolgte in Anlehnung an Arbeitsweisen der Grounded Theory ${ }^{7}$ nach Strauss und Corbin (Strauss, 1998; Strauss \& Corbin, 1996). Nachstehend sollen ihre zentralen Prinzipien sowie deren methodische Umsetzung in Grundzügen skizziert und im Zuge dessen der Rückgriff auf dieses Verfahren in Ansätzen begründet werden.

Die Methodologie der Grounded Theory zählt zu den am frühesten entwickelten, elaboriertesten qualitativen Verfahren und findet in der rekonstruktiven Sozialforschung häufig Anwendung. Sie wurde von Glaser und Strauss (1967) begründet und im Laufe der Jahrzehnte verschiedentlich weiterentwickelt. ${ }^{8}$ Dabei ging es zunächst in erster Linie um eine Abgrenzung von den damals in den Sozialwissenschaften vorherrschenden Paradigmen: Zum einen von der sogenannten „Grand Theory“ (Mills, 1959) als abstrakte soziologische Theorie mit geringer Anbindung an die Empirie, zum anderen von den etablierten standardisierten Verfahren, deren Bezug zu formalen Theorien wenig ausgeprägt war. Das zentrale Anliegen der Methodologie der Grounded Theory besteht demgegenüber in einer Verschränkung von empirischer Forschungsarbeit und Theoriebildung zur Überwindung der Spaltung von Theorie und empirischer Forschung. Sie ist auf die Entwicklung von theoretischen Konzepten aus empirischen Daten gerichtet, zielt also primär auf die Generierung einer gegenstandsbezogenen, im jeweiligen Untersuchungsfeld begründeten Theorie ab (Glaser \& Strauss, 1967; Hülst, 2010; Przyborski \& WohlrabSahr, 2010). Das bedeutet, dass Konzepte und Hypothesen primär aus dem erhobenen Material selbst entwickelt und (möglichst) nicht bereits vor der Analyse formulierte theoretische Annahmen gewissermaßen ,,von außen“

7 Ist im Folgenden von Grounded Theory die Rede, so ist hiermit stets das methodologische Verfahren, nicht die gegenstandsverankerte Theorie als Ziel bzw. erwünschtes Resultat der Forschungsmethode gemeint (zur Unterscheidung siehe z. B. Hülst, 2010; Truschkat, Kaiser \& Reinartz, 2005).

8 Auf Parallelen und Differenzen zwischen den unterschiedlichen Varianten in ihrer Konzeption und Anwendung kann an dieser Stelle nicht eingegangen werden (siehe hierzu z. B. Kelle, 2007). 
an es herangetragen werden. Insofern findet eine besondere Berücksichtigung des jeweiligen Untersuchungsbereichs mit seinen, die Aufmerksamkeit erregenden Spezifika statt, was einen weitestgehend offenen Zugang zum Forschungsfeld ermöglicht. Dies ist gerade bei wenig beforschten und tabuisierten Untersuchungsgebieten wie dem hier vorliegenden von Vorteil. Im Mittelpunkt des Erkenntnisinteresses steht dabei grundsätzlich nicht lediglich das Verstehen der subjektiven Sichtweisen der handelnden Subjekte, sondern auch das der ihnen unterliegenden Phänomene und somit der sozialen Situationen und symbolisch vermittelten Handlungszusammenhänge des Alltags (Böhm, 1994; Hülst, 2010; Strauss, 1998; Strauss \& Corbin, 1996).

Eine Stärke der Grounded Theory im Vergleich zu anderen qualitativen Verfahrensweisen liegt darin, dass sie Richtlinien zur Organisation verschiedener Phasen im Forschungsprozess bereitstellt und somit eine Strukturierung der gesamten Forschungsarbeit ermöglicht: der Datenerhebung durch ein spezifisches Samplingverfahren (das oben bereits erwähnte Theoretical Sampling), des Auswertungsprozesses durch ein bestimmtes Kodierungsparadigma. Dabei sind die Datenerhebung und -interpretation stets aufeinander bezogen, das heißt, sie finden nicht - wie insbesondere im quantitativen Forschungsparadigma - nacheinander, sondern in einem ständigen Wechselprozess statt. Es werden also Interviews geführt, ausgewertet und auf Basis eines fortwährenden Vergleichs neue zu untersuchende Fälle ausgewählt, was wiederum eine gegenstandsbezogene Verankerung der Forschung und Weiterwicklung der sich bildenden Hypothesen fördert (Hülst, 2010; Przyborski \& Wohlrab-Sahr, 2010).

Im Mittelpunkt der Interpretation steht das Kodieren des Materials, mit dem eine Überführung der empirischen Daten zunächst in Konzepte, dann in abstraktere Kategorien stattfindet. Es bezeichnet also den analytischen Prozess der Entwicklung von Konzepten, die auf einzelne Zusammenhänge verweisen, und ihrer Verdichtung zu Kategorien, die Zusammenhänge zwischen den Konzepten erfassen. Aus den abstrakteren Kategorien wird schließlich eine Theorie gebildet. Zur Loslösung von den konkreten Daten wird das Material durch Fragen aufgebrochen und neu zusammengesetzt. Dabei gilt es zu beachten, dass es beim Kodieren nicht um eine Paraphrasierung oder deskriptive Zusammenfassung des Materialinhalts geht, sondern vielmehr um die Bestimmung von Zusammenhängen und der den subjektiven Sichtweisen unterliegenden Phänomene. Somit rücken Interaktionsbedingungen, -strategien und -konsequenzen ins Zentrum der Betrachtung. Allgemein werden in der Grounded Theory drei Formen des Kodierens unterschieden, die zwar aufeinanderfolgende Schritte im Inter- 
pretationsverlauf darstellen, doch zugleich wechselseitig aufeinander bezogen sind. Es handelt sich hierbei um das offene, das axiale und das selektive Kodieren (Böhm, 1994; Przyborski \& Wohlrab-Sahr, 2010; Strauss, 1998; Strauss \& Corbin, 1996).

Bei der hier durchgeführten Auswertung der Interviewtranskripte fand eine Orientierung an den beiden ersten Kodierformen statt. Konkret gestaltete sich der Interpretationsprozess folgendermaßen: Die Auswertung begann in Anlehnung an das offene Kodieren, das auch im weiteren Verlauf stets als Einstieg in die Analyse des sukzessiv erhobenen Materials diente und somit den gesamten Forschungsprozess begleitete. Hier wird das Interviewmaterial in einer extensiven, sequenziellen Analyse durch verschiedene an den Text herangetragene Fragen aufgebrochen. Ziel ist die Loslösung von den konkreten Daten und eine Überführung dieser in erste, vorläufige Konzepte (Böhm, 1994; Przyborski \& Wohlrab-Sahr, 2010; Strauss, 1998). Im Zuge des offenen Kodierens wurde jedes Interviewtranskript vom Anfang bis zum Ende bearbeitet, dabei in verschiedene Sinnabschnitte gegliedert und interpretiert. Nach und nach fand eine Verdichtung des Materials zu Begriffen (im Sinne erster Konzepte) statt. Hierfür wurden allgemeine Fragen an das Material gestellt, welche die inhaltliche, die sprachliche sowie die interaktive Ebene in den Blick nehmen. Den Ausgangspunkt bildete auf der Inhaltsebene die Frage „Was wird erzählt?", mit der thematische Relevanzsetzungen der Interviewten sowie inhaltliche Auffälligkeiten in den Narrativen herausgearbeitet wurden. Auf der sprachlich-strukturellen Ebene zielte die Fragestellung ,Wie wird etwas erzählt?" auf die Analyse der individuellen Erzählweise mit ihren je spezifischen Charakteristika wie der semantischen Struktur und des Aufbaus der Darstellung. Die daran anschließende Frage „Wie interagieren die Interviewerin und die bzw. der Befragte miteinander?" betrachtet die Beziehungs- und Interaktionsdynamik sowie das Erzähl- und Hörerverhalten im Interview.

Im weiteren Verlauf der Interpretation wurde für die in diesem Sammelband präsentierte Analyse insbesondere auf Sinnabschnitte fokussiert, die einen direkten oder indirekten Bezug zum Umgang mit den sexuellen Missbrauchserfahrungen aufweisen. Die leitende Fragestellung lautete „Wie deuten und verarbeiten die Interviewten den sexuellen Missbrauch?". Dabei ging es bei der Rekonstruktion von subjektiven Erlebnisund Deutungsweisen des Geschehenen auch darum, die ihnen unterliegenden Phänomene sichtbar zu machen. Hiermit rücken vor allem die durch die katholische Kirche innerhalb einer religiösen Sozialisation vermittelten Wert- und Glaubensvorstellungen sowie allgemeine Charakteristika von 
Religion und deren Einfluss auf die Umgangsformen mit dem Erlebten in den Blick.

Mit Bezug auf die verschiedenen Fragen bzw. Betrachtungsebenen wurden im Zuge des offenen Kodierens für jeden Fall einige zentrale Interviewpassagen (sog. Ankerstellen) ermittelt und anschließend einer genaueren Analyse unterzogen. Diese erfolgte größtenteils im Rahmen einer im KFN gegründeten Interpretationsgruppe. An den wöchentlich stattfindenden Sitzungen nahmen Mitarbeiterinnen des Instituts sowie Forschungspraktikantinnen und -praktikanten teil. Die Interpretinnen und Interpreten entstammten verschiedenen Fachrichtungen (Psychologie, Soziologie, Kulturwissenschaften, Jura), was einen interdisziplinären Austausch ermöglichte und eine Vielzahl unterschiedlicher Perspektiven auf das analysierte Material gewährleistete. Die aus der Gruppeninterpretation gewonnenen Deutungsansätze, Lesarten und Ideen zu den jeweiligen Ankerstellen wurden möglichst umfassend protokolliert und zentrale Erkenntnisse in Memos festgehalten.

Das Verfassen von Memos spielte auch darüber hinaus eine wichtige Rolle während des gesamten Forschungsprozesses. Neben (ersten) Ergebnissen wurden in ihnen Gefühle, Eindrücke, Einfälle und Gedanken, die im Zuge der Erhebung und Auswertung des Materials auftraten, notiert. Das Schreiben solcher Notizen hatte dabei mehrerlei Funktionen: Zum einen diente es der Aktualisierung, Differenzierung und Weiterentwicklung der durch das Kodieren ermittelten Erkenntnisse. Zum anderen half es bei der Reflexion von emotionalen Reaktionen und erleichterte eine Distanzierung vom Material. In der einschlägigen Literatur wird ebenfalls darauf verwiesen, dass der Forschungsprozess in den Memos begleitet und reflektiert, ferner der Verlauf der Theoriegenerierung dokumentiert und vorangetrieben wird (Böhm, 1994; Przyborski \& Wohlrab-Sahr, 2010; Strauss, 1998; Strauss \& Corbin, 1996).

Im Fortgang der Analyse wurden die während des offenen Kodierprozesses gebildeten vorläufigen Konzepte und Interpretationsansätze zum Umgang mit sexuellen Missbrauchserfahrungen immer wieder miteinander verglichen und teils bestätigt, reformuliert oder verworfen. Hieraus resultierte eine Reduktion der anfänglichen Fülle an Begriffen und Ideen sowie eine Verdichtung und Verfeinerung der einzelnen Konzepte. Nach dieser vergleichenden Überprüfung und Modifikation der ersten Konzepte und Interpretationsansätze wurden diese in Zusammenhang zueinander gestellt und danach zu Kategorien gruppiert. Dieser Verdichtungsvorgang markiert den Übergang zum axialen Kodieren, das der Ausarbeitung von Kategorien (und Subkategorien) dient. Im Zuge dessen werden die einzel- 
nen Kodes ${ }^{9}$ in Hinblick auf ihre Relationen zueinander intensiver analysiert: Zum einen wird das Verhältnis der Konzepte zu den Kategorien, zum anderen die Beziehung zwischen den verschiedenen Kategorien (und ihren Subkategorien) ausgelotet. Das Kodieren befasst sich hierbei mit der „Achse“ einer Kategorie, um jeweils wechselseitige Zusammenhänge zu anderen Kategorien zu ermitteln (Böhm, 1994; Przyborski \& WohlrabSahr, 2010; Strauss, 1998).

Im gesamten Interpretationsprozess und insbesondere beim axialen Kodieren spielte demnach der permanent stattfindende, wechselseitige Vergleich zwischen Konzepten und Kategorien eine entscheidende Rolle. Dieser vollzog sich zunächst auf der Ebene des einzelnen Falls und in einem zweiten Schritt in fallübergreifender Perspektive, also zwischen den 15 Fällen. Im Zentrum des Vergleichs standen Übereinstimmungen bzw. Ähnlichkeiten sowie Unterschiede innerhalb der und zwischen den Kodes gemäß dem Prinzip einer jeweils minimalen und maximalen Kontrastierung.

Im Rahmen der hier vorgelegten Auswertung zielte die Kodierung des Interviewmaterials auf die Rekonstruktion von kontrastierenden Deutungsmustern und mit ihnen zusammenhängenden Verarbeitungsversuchen der sexuellen Missbrauchserfahrungen ab. Hierzu wurde mittels der skizzierten Analyseschritte zunächst die Varianz des Untersuchungsfeldes ausgelotet. Die anfängliche Bildung einer Vielzahl an Konzepten zum Umgang mit dem Erlebten lieferte in diesem Zusammenhang einen umfassenden und detaillierten Überblick über das Phänomen und seine Ausprägungen. Sodann erfolgte im Laufe des weiteren Interpretationsprozesses eine Verdichtung der verschiedenen im Material gefundenen Umgangsformen zu fünf Kategorien, unter die sich die unterschiedlichen Reaktionsweisen gruppieren ließen. Sie stellen typische Deutungs- und Verarbeitungsmuster der interviewten Betroffenen dar und bilden das Spektrum an unterschiedlichen und einander ähnelnden Formen des Umgangs mit den sexuellen Missbrauchserfahrungen im vorliegenden Sample ab. ${ }^{10}$

9 Der Begriff Kode ist eine Sammelbezeichnung für Konzepte und Kategorien (Przyborski \& Wohlrab-Sahr, 2010).

10 Eine detaillierte Beschreibung und Analyse der herausgearbeiteten biografischen Umgangsformen findet sich in Kapitel 5 dieses Sammelbands (Fernau, 2014). 


\section{Literatur}

Bergmann, C. (2011). Abschlussbericht der Unabhängigen Beauftragten zur Aufarbeitung des sexuellen Kindermissbrauchs, Dr. Christine Bergmann. Berlin: Geschäftsstelle der Unabhängigen Beauftragten zur Aufarbeitung des sexuellen Kindesmissbrauchs.

Böhm, A. (1994). Grounded Theory. Wie aus Texten Modelle und Theorien gemacht werden. In A. Böhm, A. Mengel \& T. Muhr (Hrsg.), Texte verstehen. Konzepte, Methoden, Werkzeuge (S. 121-140). Konstanz: UVK.

Bundschuh, C. (2010). Sexualisierte Gewalt gegen Kinder in Institutionen. Nationaler und internationaler Forschungsstand. München: Deutsches Jugendinstitut.

Derogatis, L. R. \& Melisaratos, N. (1983). The Brief Symptom Inventory: An introductory report. Psychological Medicine, 13, 595-605.

Egle, U. T. \& Abhary, S. G. (2005). Sexueller Missbrauch, Misshandlung, Vernachlässigung, Erkennung, Therapie und Prävention der Folgen früher Stresserfahrungen. Stuttgart: Schattauer.

Franke, G. H. (2000). Brief Symptom Inventory von L. R. Derogatis (Kurzform der SCl-90-R) - Deutsch Version. Göttingen: Beltz Test.

Glaser, B. G. \& Strauss, A. L. (1967). The discovery of grounded theory: Strategies for qualitative research. Chicago, IL: Aldine.

Häuser, W. \& Schiedermaier, P. (2005). Das Fibromyalgiesyndrom in der Sozialgerichtsbarkeit - Psychosoziale Risikofaktoren und Praediktoren der Inanspruchnahme medizinischer Leistungen. Psychotherapie, Psychosomatik, Medizinische Psychologie, 55, 72-78.

Helfferich, C. (2009). Die Qualität qualitativer Daten: Manual für die Durchführung qualitativer Interviews (3., überarbeitete Auflage). Wiesbaden: VS.

Hellmann, D. F. \& Bartsch, T. (2014). Berücksichtigung der Belange von Betroffenen sexuellen Missbrauchs im Opferentschädigungsgesetz: Gleiches Recht für alle? Monatsschrift für Kriminologie und Strafrechtsreform, 97, 131-149.

Höcker, A. \& Mehnert, A. (2012). Posttraumatische Belastung bei Krebspatienten: Validierung der deutschen Version der Posttraumatic Stress Disorder Checklist Civilian Version (PCL-C). Zeitschrift für Medizinische Psychologie, 21, 68-79.

Hopf, C. (1978). Die Pseudo-Exploration: Überlegungen zur Technik qualitativer Interviews in der Sozialforschung. Zeitschrift für Soziologie, 7, 97-115.

Hopf, C. (2000). Forschungsethik und qualitative Forschung. In U. Flick, E. von Kardorff \& I. Steinke (Hrsg.), Qualitative Forschung: Ein Handbuch (S. 589-600). Reinbek: Rowohlt.

Hülst, D. (2010). Grounded Theory. Verfügbar unter: http://www.fallarchiv.unikassel.de/wp-content/uploads/2011/02/huelst_grounded_theory2.pdf

John Jay College (2004). The nature and scope of sexual abuse of minors by Catholic priests and deacons in the United States, 1950-2002. Washington, DC: United States Conference of Catholic Bishops (USCCB). 
Kelle, U. (2007). „Emergence“ vs. „forcing“ of empirical data? A crucial problem of „Grounded Theory“ reconsidered. In G. Mey \& K. Mruck (Eds.), Grounded Theory Reader (pp. 133-156). Köln: Zentrum für historische Sozialforschung.

Kelle, U. \& Kluge, S. (1999). Vom Einzelfall zum Typus: Fallvergleich und Fallkontrastierung in der qualitativen Sozialforschung. Opladen: Leske + Budrich.

Kleining, G. (1982). Umriss zu einer Methodologie qualitativer Sozialforschung. Kölner Zeitschrift für Soziologie und Sozialpsychologie, 34, 224-253.

Kruse, J. (2014). Qualitative Interviewforschung: Ein integrativer Ansatz. Weinheim: Beltz.

Lucius-Hoene, G. \& Deppermann, A. (2002). Rekonstruktion narrativer Identität. Ein Arbeitsbuch zur Analyse narrativer Interviews. Opladen: Leske + Budrich.

Lueger-Schuster, B. (2012). Psychotraumatologische Fragestellungen zu Gewalt und Missbrauch in der Katholischen Kirche. Wien: Universität Wien.

Merton, R. K., Fiske, M. \& Kendall, P. L. (1956). The focused interview: A manual of problems and procedures. Glencoe, IL: Free Press.

Mills, C. W. (1959). The sociological imagination. New York, NY: OUP.

Przyborski, A. \& Wohlrab-Sahr, M. (2010). Qualitative Sozialforschung. Ein Arbeitsbuch. München: Oldenbourg.

Sanderson, C. (1990). Counselling adult survivors of child sexual abuse. London, UK: Kingsley.

Schumacher, J., Leppert, K., Gunzelmann, T., Strauß, B. \& Brähler, E. (2005). Die Resilienzskala - Ein Fragebogen zur Erfassung der psychischen Widerstandsfähigkeit als Personmerkmal. Zeitschrift für Klinische Psychologie Psychiatrie und Psychotherapie, 53, 16-39.

Schütze, F. (1976). Zur soziologischen und linguistischen Analyse von Erzählungen. In G. Dux \& T. Luckmann (Hrsg.). Internationales Jahrbuch für Wissens- und Religionssoziologie, Band 10 (S. 7-41). Opladen: Westdeutscher Verlag.

Schütze, F. (1978). Die Technik des narrativen Interviews in Interaktionsfeldstudien Dargestellt an einem Projekt zur Erforschung von kommunalen Machtstrukturen. Bielefeld: Universität Bielefeld.

Schütze, F. (1983). Biographieforschung und narratives Interview. Neue Praxis, 3, 283-293.

Straus, M. A. (1979). Measuring intrafamily conflict and violence: The Conflict Tactics (CT) Scales. Journal of Marriage and the Family, 41, 75-88.

Strauss, A. L. (1998). Grundlagen qualitativer Sozialforschung. Datenanalyse und Theoriebildung in der empirischen soziologischen Forschung (2. Auflage). München: Wilhelm Fink.

Strauss, A. L. \& Corbin, J. (1996). Grounded Theory. Grundlagen qualitativer Sozialforschung. Weinheim: Beltz.

Teegen, F. (1997). Deutsche Übersetzung der Posttraumatic Stress Disorder Checklist (PCL-C) des National Center for PTSD. Hamburg: Universität Hamburg.

Truschkat, I., Kaiser, M. \& Reinartz, V. (2005). Forschen nach Rezept? Anregungen zum praktischen Umgang mit der Grounded Theory in Qualifikationsarbeiten. 
Forum Qualitative Sozialforschung, 6. Verfügbar unter http://nbnresolving.de/urn: nbn:de:0114-fqs0502221.

Wetzels, P. (1997). Gewalterfahrungen in der Kindheit: Sexueller Mißbrauch, körperliche Mißhandlung und deren langfristige Konsequenzen. Baden-Baden: Nomos.

Zimmer, A., Lappehsen-Lengler, D., Weber, M. \& Götzinger, K. (2014). Sexueller Kindesmissbrauch in kirchlichen Institutionen - Zeugnisse, Hinweise, Prävention. Weinheim: Beltz. 
